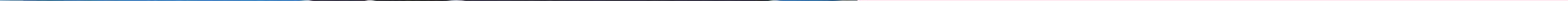




\title{
ESTRATEGIAS METACOGNITIVAS Y COMPRENSIÓN LECTORA EN UNIVERSITARIOS MIEMBROS DE COMUNIDADES INDÍGENAS
}

\section{Metacognitive strategies and reading comprehension in universitary students members of indigenous communities}

Fecha de recepción: 17 de marzo de 2013 - Fecha de aprobación: 10 de junio de 2013

\begin{abstract}
RESUMEN
Son escasos los estudios realizados sobre estrategias metacognitivas y compresión lectora en estudiantes indígenas, y menos aún en población universitaria. Este estudio descriptivo buscó identificar y analizar las estrategias metacognitivas usadas en relación con la comprensión lectora durante actividades estructuradas, con el fin de generar unas recomendaciones académicas generales para el programa institucional. El diseño metodológico incluyó informes verbales, entrevistas, observación y cuestionarios para indagar sobre el uso de las estrategias metacognitivas de los universitarios. Participaron un grupo de 7 estudiantes miembros de comunidades indígenas, 3 mujeres y 4 hombres, en edades comprendidas entre 17 y 22 años. Se encontró que los participantes conocen las estrategias metacognitivas que permiten mejorar el nivel de comprensión lectora, aunque presentan dificultad en la planificación, supervisión y evaluación de estas. Se encontraron rasgos que denotan procesos de adaptación a una nueva cultura académica. Los resultados evidenciaron un cambio y una adaptación a nuevas culturas académicas, que traen consigo una serie de retos y dificultades que se reflejan en el rendimiento académico.
\end{abstract}

PALABRAS CLAVES

Metacognición, Estrategias Metacognitivas, Comprensión Lectora, población indígena.

\section{ABSTRACT}

Studies of metacognitive strategies in relation to reading comprehension in college Indians students are very few. However, there are studies and research in basic secondary students. What drove this research in the university population to expand speech therapy practice. Identify and analyze students' metacognitive strategies in indigenous members of the Universidad Nacional de Colombia in relation to reading comprehension during structured activities. We conducted a descriptive study in which we used: verbal reports, interviews, observation and questionnaires to investigate the use of metacognitive strategies of Indian students. The study included a sample of 7 student members of indigenous communities, 3 women and 4 men, aged between 17 and 22 . It was found that the participants know the metacognitive strategies that improve reading comprehension level, but have difficulty in planning, monitoring and evaluation of these. Features were found which denote processes of adaptation to a new academic culture. The results are evidence of change and adaptation to new academic cultures, which brings a number of consequences that are reflected in academic performance.

KEYWORDS

Metacognition, Metacognitive Strategies, Reading Comprehension, Basic Cognitive Processes. 
L a actividad metacognitiva se entiende como la capacidad que se tiene para autorregular el aprendizaje, vista como una estrategia para mejorar los procesos cognitivos (percepción, atención, memoria, comunicación, imaginación, comprensión y lectura) a través del buen manejo de los recursos mentales que se poseen (Ladino y Tovar, 2005).

La metacognición es uno de los mecanismos para el conocimiento de sus propios procesos y contenidos mentales que incluye estrategias de control y conocimiento (Flavell, 2002). El control incluye las actividades cognitivas que implican planificación, supervisión del proceso intelectual y evaluación de los resultados; y el conocimiento, se entiende como la habilidad para tomar conciencia del funcionamiento de nuestra manera de aprender.

Para la comprensión de este planteamiento, Flavell (2002) propone el Modelo de Monitoreo Cognitivo que contiene cuatro componentes: conocimiento metacognitivo, experiencia metacognitiva, tipo de tarea y estrategias. Del mismo modo, Baker, Brown, Antonijevic y Chadwick, (1981), plantean que en el proceso de control metacognitivo intervienen tres etapas las cuales son fundamentales en la realización de diversas tareas, dichas etapas son planificación, supervisión y evaluación.

La Planificación. Implica reconocer la naturaleza de la tarea, conocimiento del dominio que se tiene sobre una tarea, para poder relacionar de forma sencilla la información nueva con aquella que le parece relevante (aprendizaje significativo) y por último, establecimiento de objetivos a corto plazo, para decidir sobre qué estrategias utilizará para ello.

La supervisión. En esta fase, el estudiante es autorregulador de su aprendizaje y constantemente se monitorea sobre cómo está aprendiendo, y si está utilizando las estrategias adecuadas.

La evaluación. Implica que el estudiante sea consciente de cuánto, cómo, en qué tiempo aprendió y con qué tipo de dificultades se encontró (Sánchez y Maldonado, 2008).

En el contexto universitario leer es un proceso estratégico ya que está encaminado a recabar cierto conocimiento de un texto según el propósito de lectura que autorregula la actividad cognitiva del lector (Carlino, 2005). Como actividad mental guiada por el pensamiento, la lectura implica un sistema de procesos que va más allá de la traducción de símbolos gráficos a modos de lenguaje oral e interpretación de su significado manifiesto o literal que pone en relación a los conceptos, ideas y valores que lo contienen. Generalmente, la lectura en la educación superior responde únicamente al reconocimiento del contenido de la lectura (Carlino, 2005) donde los estudiantes deben saber qué dice el texto, sin tener un propósito específico y sin responder estratégicamente a un aprendizaje significativo.

Como proceso dinámico e interactivo, la lectura involucra un conjunto de operaciones cognitivas a través de las cuales el lector va estableciendo relaciones entre las diferentes ideas (proposiciones del mismo (Tapia y Luna, 2008).

La comprensión de textos es un proceso más complejo que exige del lector, al menos, tres importantes tareas: la extracción del significado; la integración de ese significado en la memoria y la elaboración de las inferencias necesarias para una comprensión plena (Cuetos, et al, 1996 citado en Jiménez, 2004).

El lector estratégico debe tener un objetivo al leer un texto y, por ello, cuando se promueve la lectura es fundamental que vayan haciendo preguntas desde el inicio del proceso. Estos aspectos metacognitivos de la comprensión son los que permiten que la lectura sea significativa, para que el lector se haga consciente de qué quiere leer, cómo lo logra, qué hace para comprender y si realmente está leyendo comprensivamente, implementando así, sus estrategias metacognitivas (Inga, 2007).

Los aspectos metacognitivos de la lectura, incluyen el control de destrezas cognitivas apropiadas, en el sentido de planificar actividades cognitivas, elegir entre varias actividades alternativas, supervisar la ejecución de las actividades elegidas y cambiar las actividades si fuera preciso, lo mismo que evaluar si se han conseguido los objetivos propuestos al comenzar la tarea, así como la comprensión misma. Además, para ser hábil en metacomprensión hay que tener presente la interacción que se da entre la persona (en este caso el lector), la tarea (el objetivo que tiene el lector ante la lectura), las estrategias y el texto (Montenegro, 1996 citado en Jiménez, 2004).

En el ingreso a la educación superior algunos estudiantes experimentan cambios en su concepción de aprendizaje y en la toma de conciencia (metacognición) de la importancia de aprender más y mejor (Martínez, 2004). Carlino (2005), plantea que existen diferentes modos de leer y comprender escritos, modos que forman parte de diversas culturas lectoras ya que las dificultades para comprender lo que se lee en la universidad no se debe a la carencia de un habilidad o técnica elemental sino que al ingresar a los estudios superiores se ven enfrentados a nuevas culturas escritas que corresponden a los distintos campos de estudio, lo que los obliga a cambiar su identidad como pensadores y analizadores de textos convirtiéndose en lectores modelo (Carlino, 2005).

Los estudiantes, como es el caso de miembros de comunidades indígenas que ingresan a la Universidad Nacional, se enfrentan a una nueva cultura académica asumiendo diversos retos; el primero de ellos, es el cambio en normas y valores de comportamiento que se exigen pero no se enseñan explícitamente. El segundo lugar, los textos exponen saberes que los estudiantes no conocen y/o tienen, pues sus contenidos están dirigidos a expertos en el tema.

Adicionalmente, los estudiantes son evaluados en función de un determinado modelo de lectura que aun no se ha adquirido (Carlino, 2005). Todo lo anterior confirma, que los estudiantes no son más que inmigrantes que se enfrentan a una cultura nueva asumiendo una serie de cambios para iniciar un proceso de adaptación, que les permitirá ser miembros de una cultura simbólica nueva, invalidando así la postura errónea de que los estudiantes de otras 
culturas presentan dificultades de aprendizaje. Las diferencias no son deficiencias. Los patrones culturales no son comparables. Cada patrón de aprendizaje cultural es legítimo en sí mismo.

Las habilidades que garantizan un buen lector son de dos tipos: cognitivas, que permiten procesar la información del texto, y las metacognitivas, que permiten tener conciencia del proceso de comprensión y controlarlo a través de actividades de planificación, supervisión y evaluación. (Puente, 1994 citado en Jiménez, 2004). En el mismo contexto de la metacognición y la comprensión lectora, existe la necesidad de relacionar los procesos cognitivos básicos con las actividades mentales implícitas en la integración de la información que proporciona la cultura escrita.

El primer proceso cognitivo básico es la memoria que es la capacidad de almacenar y recuperar información. Desde la psicología cognitiva se han definido varios tipos de memoria, una de ellas es la memoria de trabajo, considerada como un sistema encargado de almacenar y administrar transitoriamente toda la información (de distintas modalidades) que se encuentra actualmente en uso para la realización de una tarea específica (Carrillo-Mora, 2010).

La atención es la capacidad de seleccionar, focalizar y filtrar la información sensorial para dirigir los procesos mentales. La selección depende de las características del estímulo, de las necesidades, las experiencias y las demandas del medio. La focalización implica resistencia a la distracción, conservando la habilidad para establecer el foco de atención, mantenerlo y cambiarlo por uno nuevo si la situación lo exige. Por ultimo, se encuentra la filtración que es la capacidad de anular distractores irrelevantes manteniendo la concentración en el estímulo relevante. De otra parte, la percepción es la capacidad de organización y análisis de la información que provee el ambiente, interpretando el estímulo como objeto significativo (Gallegos y Gorostegui, 2002).

Los proceso cognitivos básicos, en cierta medida involucran habilidades del lenguaje (contenido, forma y uso), inmersas en competencias comunicativas que posibilitan procesos de aprendizaje positivos, los cuales inciden en un desempeño académico de calidad (Flórez y Moreno, 2004).

A raíz del mandato de la constitución política de 1991, la Universidad del estado, se ha interesado por incluir poblaciones excluidas de la educación superior, es por esto que el Consejo Superior Universitario (1986) crea el Acuerdo 018 que estableció el Programa de Admisiones Especiales para Bachilleres de Comunidades Indígenas con el objetivo de brindar oportunidades a miembros de poblaciones indígenas y así facilitarles el acceso a la educación superior. Este acuerdo permitió el acceso e inclusión a la educación superior por parte de comunidades marginadas (Consejo Superior Universitario, 1999).

Desde la Fonoaudiología, se considera necesario establecer programas que permitan mejorar las capacidades cognitivas, lingüísticas y comunicativas de las personas. Por tal razón, se creó el Programa de Análisis de las Estrategias Metacognitivas en relación con la Comprensión Lectora para facilitar la inclusión e integración de los estudiantes miembros de comunidades indígenas en diferentes ambientes académicos. Se evidenció la necesidad de desarrollar programas como este para disminuir la deserción, el fracaso y el bajo rendimiento académico por causa de la dificultad que se presenta en la comprensión de lectura; lo que permite generar un impacto social y contribuir con la inclusión de esta población en la educación superior.

\section{METODO}

El presente estudio de naturaleza exploratoria-descriptiva tuvo como propósito observar, describir, caracterizar y contrastar el comportamiento metacognitivo de los estudiantes frente a la comprensión de lectura en actividades estructuradas.

Se convocó al universo de 23 estudiantes que constituyen toda la población de procedencia indígena universitaria y se trabajó con quienes aceptaron participar voluntariamente. El estudio se desarrolló con un grupo de 7 estudiantes, miembros de comunidades indígenas: 3 mujeres y 4 hombres, en edades comprendidas entre 17 y 22 años. La procedencia de los participantes fue de las regiones de Nariño, Orinoquía y Costa Atlántica; de estratos socioeconómicos 1 y 2.

Con relación a las carreras cursadas, 4 estudiantes eran de Ingeniería, 2 de Economía y 1 de Medicina. Todos firmaron el consentimiento informado en el que aceptaron la aplicación de la instrumentación del estudio.

Se realizó un taller introductorio en el que se recordó el objetivo del estudio y las características del programa y sus beneficios. El programa se desarrolló en 8 semanas, cada sesión tuvo una duración de 4 horas. Una vez terminada cada sesión se analizó la información obtenida para una posterior interpretación global de los resultados.

Para la recolección de la información se utilizaron varios instrumentos tales como: formatos de entrevista para recolectar datos generales y socio demográficos, Encuesta de habilidades metacognitivas, basada en el Cuestionario de Actividad Metacognitiva y Prueba de Comprensión Lectora (Cerchiaro, Arbeláez, y Herrera, 2009). Esta prueba buscó conocer la percepción sobre la actividad metacognitiva y nivel de comprensión lectora en estudiantes universitarios.

Se usó también la encuesta de habilidades de lectura, basada en los siete rasgos para evaluar la comprensión de lectura de las Pruebas de Comprensión Lectora y Producción de Textos (CL-PT) (Gajardo y Medina, 2010). Los siete rasgos evaluados que permitieron identificar la autopercepción sobre sus habilidades lectoras, fueron: comprensión de estructuras textuales, comprensión literal, comprensión inferencial, comprensión critica, comprensión metacognitiva, reorganización de la información y manejo del código.

Como se anotó, para observar las habilidades metacognitivas que los estudiantes universitarios usaban frente a la lectura se aplicaron escalas adaptadas de conciencia lectora (Cerchiaro, Arbeláez, y Herrera, 2009), además de observación directa sobre la población en actividades e informes verbales que incluían ítems de comprensión, elaboración de síntesis y auto percepción de sus habilidades metacognitivas en lectura, se realizó una observación detallada du- 
rante una actividad que permitió identificar el nivel de comprensión literal e inferencial de la información presentada en los textos.

Los datos obtenidos se analizaron mediante un proceso de análisis cualitativo, que tuvo como fin clasificar por medio de categorías, el uso de las estrategias metacognitivas en relación con el conocimiento y control en la comprensión lectora y los procesos cognitivos básicos.

\section{RESULTADOS}

A partir del análisis cualitativo realizado se encontraron los siguientes resultados del comportamiento del grupo de 7 estudiantes participantes.

\section{Procesos Cognitivos Básicos.}

\section{Atención.}

Los estudiantes presentaron atención focalizada, al lograr resistirse a la distracción, centrando su atención en una sola fuente de información, manteniendo el nivel de concentración que exigía la actividad.

\section{Memoria.}

Los estudiantes usan los procesos de codificación, almacenamiento y recuperación de la información. Se observó una capacidad más limitada al no organizar y categorizar los elementos para lograr almacenarlos y recuperarlos posteriormente.

\section{Percepción.}

Los estudiantes identifican los aspectos más evidentes de las imágenes, imponiendo su propia estructura de lo que ven. Integran la información percibida en un conjunto significativo según sus conocimientos previos y su actitud subjetiva. Le dan valor a lo que se desea ver y no a la realidad del estímulo.

\section{Estrategias Metacognitivas.}

Los resultados fueron agrupados según las dos categorías del Modelo de Monitoreo Cognitivo de Flavell: conocimiento metacognitivo y control metacognitivo.

\section{Conocimiento Metacognitivo en Lectura.}

A partir de los elementos que conforman la variable personal del conocimiento metacognitivo, se observó que los estudiantes muestran un nivel de resistencia a la participación en actividades de socialización, previas a una tarea específica.

En la autopercepción de su conocimiento metacognitivo, los estudiantes coinciden en afirmar que es importante a la hora de desarrollar una actividad, atribuirle la importancia que ésta merece.

Respecto a la variable de tarea se encontró que en actividades de lectura las características mas relevantes son: la identificación del tipo de texto; dentro del texto reconocen secuencias de relaciones, causa-efecto, juicios de realidad y fantasía, hechos de opiniones e identifican las características y detalles de los personajes, además reconocen y usan adecuadamente los conectores, preposiciones y conjunciones. En su mayoría los estudiantes coinciden en decir que al llevar a cabo cualquier actividad, la eficacia de ésta depende del nivel de atención y esfuerzo que se le dedique.

Frente a la variable de estrategia se observó que los estudiantes identificaron cuáles son los objetivos de las tareas asignadas, y utilizaron distintas estrategias de aquellas que les permitieron tener éxito en sus actividades académicas: estrategias mnemotécnicas (71 $\%$ ), de asociación semántica (57\%) y de selección de información relevante (71\%). También lograron llevar a cabo procedimientos, adaptándose a reglas y nuevas estrategias que les facilita su aprendizaje.

\section{Control Metacognitivo en Lectura.}

Se observaron las variables relacionadas con el control metacognitivo según el modelo de Flavell: planificación, supervisión y evaluación de los resultados del proceso en la comprensión lectora. En la etapa de planificación se encontró que los estudiantes logran identificar la naturaleza de la tarea y los objetivos de ésta, pero realizan con dificultad el proceso que guía el alcance de sus objetivos. Al no relacionar los conocimientos previos con los estímulos presentados, se genera que los estudiantes no planteen unas estrategias apropiadas de planificación en lectura, como lo son las estrategias de pre-lectura que involucran la globalización del contenido del texto a partir del título, palabras claves, graficas e imágenes, entre otras. Sin embargo, aunque identifican la importancia de las estrategias anteriores, no logran usarlas conscientemente en su proceso lector.

Con relación a la etapa de supervisión se observó que los estudiantes no conocen las estrategias de supervisión que pueden usar para autorregular su comprensión, lo que causa que no respondieran a las estrategias de monitoreo para supervisar el proceso. Como el nivel de comprensión no era suficiente, no actuaron sobre el proceso comprensivo y no modificaron de forma consciente la estrategia natural utilizada hasta ese momento.

Por último, se encontró que en la etapa de evaluación se identificó que los estudiantes por el hecho de no seleccionar estrategias adecuadas que guían el proceso de comprensión y de regulación de la comprensión, tienen dificultad al planificar y supervisar el proceso, evidenciando que por no ser conscientes de las estrategias metacognitivas, presentan dificultad para evaluar cuándo, cómo y en qué tiempo comprendieron la información.

En síntesis, al analizar las estrategias metacognitivas de los 7 estudiantes observados, se encontró que controlaron los procesos cognitivos básicos (atención, memoria y percepción), conocen la naturaleza de las tareas presentadas y sus requerimientos en cuanto a nivel de esfuerzo, atención y relevancia, y conocen sus estrategias metacognitivas pero no logran ejecutarlas, controlarlas y evaluarlas en tareas de comprensión lectora.

\section{DISCUSIÓN}

La teoría metacognitiva expuesta inicialmente por el psicólogo John Flavell, afirma, que el conocimiento y el control de los procesos mentales son importantes en cualquier actividad cognitiva. Es fundamental que las personas conozcan y regulen sus procesos de 
adquisición de nuevos conocimientos. Teniendo en cuenta la teoría metacognitiva, los resultados muestran que los estudiantes tienen el conocimiento de las estrategias que pueden ser empleadas en actividades de comprensión lectora, pero no logran controlar dichas estrategias, por lo que requieren de un ente externo que les permita impulsar la ejecución de estrategias de control metacognitivo (planificación, supervisión y evaluación).

Este estudio confirma lo que plantea Carlino (2005), en tanto que el ingreso a nuevas culturas académicas supone una serie de cambios y retos para los estudiantes que deben ser vistos como un proceso intercultural que amerita la generación de espacios, prácticas y procesos que faciliten el ingreso a la nueva cultura sin demeritar el acervo cultural de la propia.

La comprensión lectora, como lo plantea la teoría y se reconfirma en los hallazgos de este estudio, es sin lugar a dudas una práctica educativa y cultural que amerita una meta-reflexión que de cuenta de las implicaciones para los miembros de comunidades indígenas, quienes manejan estas estrategias de manera distinta a la esperada típicamente como resultado de una cultura académica.

En síntesis, se sugiere que probablemente la adaptación a una nueva cultura escritural, como es el caso de los estudiantes indígenas, en donde deben enfrentarse a nuevos ambientes académicos y comunicativos, demanda estrategias que no han tenido entrenamientos previos suficientes para desarrollar las tareas iniciales de forma exitosa.

Sin embargo se logró advertir que al utilizar adecuadamente las estrategias de control metacognitivo, como las que se realizaron en el programa en el que participaron, el estudiante podría ser un lector estratégico, regulando su estructura metacognitiva, haciendo construcciones significativas de conocimiento en su área de forma- ción profesional y generalizando sus habilidades cognitivas en la comprensión de lectura.

\section{CONCLUSIONES.}

Los hallazgos encontrados, denotan una gran necesidad de explorar el tema, dado que antes que responder las inquietudes, lo que hacen es poner en evidencia las múltiples preguntas alrededor de la alfabetización académica. Dicho hallazgos poseen valor en términos de la necesidad de crear, establecer y promover el campo de lo que se debería reconocer como Etnofonoaudiología. Estos estudios estarían respondiendo a las necesidades fonoaudiológicas de las minorías étnicas.

Los resultados encontrados, son un llamado de atención a las políticas de discriminación positiva y admisión directa que se tienen en las universidades para estos grupos poblacionales. Se evidencia que no es suficiente la ampliación de cobertura y acceso, si no que se hace necesario mirar en detalle las implicaciones socio-culturales que se derivan de estas exposiciones a ambientes alfabetizados.

En este mismo sentido, se requiere que los docentes universitarios promuevan el aprendizaje significativo, enseñando a comprender el lenguaje propio de cada ciencia a partir de las estrategias metacognitivas en relación con la lectura en distintos ambientes y actividades académicas, para promover de esta manera la inclusión de todos los estudiantes a nuevas culturas escritas, con su respectiva apropiación del lenguaje usado en su profesión.

A los programas existentes en la Universidad Nacional de Colombia deberían incluírseles contenidos que promuevan el desarrollo de estrategias metacognitivas, no solo para estudiantes miembros de comunidades indígenas sino para los estudiantes regulares. 


\section{Referencia:}

Las referencias a otras obras son una parte

muy importante en la literatura científica;

ya que estas permiten conocer más sobre los autores y mantener vivas sus voces dentro del texto.

Baker, L., Brown, Antonijevic \& Chadwick (1981). Metacognition and reading process. Mayor, J; Suengas, A; y González J. (1995). Estrategias metacognitivas: aprender a aprender y aprender a pensar. Madrid: Editorial síntesis, S.A.

Carlino, P. (2005). Escribir, leer y aprender en la universidad: una introducción a la alfabetización académica. Buenos Aires: Fondo de Cultura Económica. 67-90.

Carrillo-Mora, P. (2010). Sistemas de memoria: reseña histórica, clasificación y conceptos actuales. Primera parte: Historia, taxonomía de la memoria, sistemas de memoria de largo plazo: la memoria semántica. Salud Mental, 33(1), 85-93

Cerchiaro, E., Arbeláez, M., Herrera, J. (2009). Cuestionario de Actividad Metacognitiva y Prueba de Comprensión Lectora. Santa Marta. Universidad del Magdalena.

Consejo Superior Universitario. (1999). Acuerdo Número 018 de 1999 (Acta 14 del 26 de julio). Universidad Nacional de Colombia.

Flavell, J.H. (2002). Cognitive development (4th ed.). Upper Saddle River, NJ: Prentice Hall.

Flórez, R. \& Moreno, M. (2004). El Lenguaje en la Educación una Perspectiva Fonoaudiológica. En: Lenguaje en la Educación Superior. Bogotá: Universidad Nacional. Unibiblos. p. 285-325.

Gajardo, A. \& Medina, A. (2010). Pruebas de Comprensión Lectora y Producción de Textos (CL-PT). Chile: Ediciones Universidad Santiago de Chile.

Gallegos. S. \& Gorostegui. M. (2002) Procesos cognitivos. Disponible en http:// www.reocities.com/sicotema/1190494636.pdf. Consultado el 31 octubre de 2011.

Jiménez, V. (2004) Metacognición y comprensión de la lectura: Evaluación de los componentes estratégicos (procesos y variables) mediante la Elaboración de una escala de conciencia Lectora (ESCOLA). Madrid. Universidad Complutense de Madrid. 2004: 89

Inga, M. (2007). Estrategias metacognitivas para la comprensión y producción de textos continuos. Investigación Educativa, vol. 11. 54 (12): 1-15. 2007. Disponible en: sisbib.unmsm.edu.pe. [consultado el 21 de octubre de 2011].

Ladino, Y. \& Tovar, J. (2005). Evaluación de las Estrategias Metacognitivas, para la Comprensión de Textos Científicos. Colombia: Enseñanza de las Ciencias. Número extra VII Congreso. 1-5. Disponible en: http://www.unne.edu. ar [consultado el 26 de febrero de 2011].

Martínez, R. (2004). Concepción de aprendizaje, metacognición y cambio conceptual en estudiantes universitarios de psicología. España: Universidad de Barcelona Facultad de Psicología Departamento de Psicología Básica.

Sánchez, C. \& Maldonado, M. (2008). Comprensión de lectura y metacognición en jóvenes, una Herramienta para el éxito en la comprensión de lectura, Estudio descriptivo exploratorio en niños de once a trece años de Edad que cursan primero de bachillerato, pertenecientes al Colegio Gimnasio los Robles de Bogotá D.C. Colombia. Universidad, Colegio Mayor de Nuestra Señora del Rosario.

Tapia, V. \& Luna, J. (2008) procesos cognitivos y desempeño lector. Universidad Nacional Mayor de San Marcos, Revista de Investigación en Psicología $11,1$. 